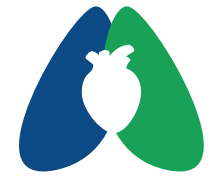

ASSOBRAFIR C I Ê N C I A

\section{Síndrome de Burnout e qualidade de vida em fisioterapeutas intensivistas do Estado de Sergipe ${ }^{a}$}

\author{
Burnout Syndrome and quality of life in intensive care \\ physiotherapists
}

Yago Alves Lima1* (D), Mariana Andrade Dantas² (D), Lucas Aragão da Hora Almeida ${ }^{3}$ (D), Fernanda Oliveira de Carvalho ${ }^{4}$ (i), Carlos José Oliveira de Matos ${ }^{4}$ (1)

\begin{abstract}
Resumo
Introdução: A Unidade de Terapia Intensiva é um ambiente considerado estressante, podendo afetar a qualidade de vida do fisioterapeuta intensivista e originar a Síndrome de Burnout. Objetivo: Avaliar a presença de Síndrome de Burnout, a qualidade de vida e a correlação com carga horária. Métodos: Estudo transversal realizado com 56 fisioterapeutas atuantes em UTIs do estado de Sergipe. Foram aplicadas duas escalas, o Medical Outcomes Short-Form Health Survey que avalia qualidade de vida e o Mas/ach Burnout Inventory que avalia a Síndrome de Burnout, e um questionário sociodemográfico. Resultados: O sexo feminino foi mais prevalente, com $80,3 \%$ da amostra, a média de idade foi 31,6 $6 \pm 5,6$ anos. Na qualidade de vida, o domínio mais afetado foi Dor com uma mediana de 62,0 pontos. Na escala para Síndrome de Burnout, a Exaustão emocional teve média de 28,9 \$5,9 pontos com pontuação correspondente a alto nível em $62,5 \%$ da amostra; a média de pontos em Realização profissional foi de 15,1 $\pm 3,7$ pontos, com alto nível de pontuação em $100 \%$ da amostra; e a Despersonalização obteve uma média de pontos de 17,05 $\pm 2,9$ com alto nível de pontuação, presente em 92,8\% dos fisioterapeutas. A qualidade de vida e a Síndrome de Burnout não se correlacionaram com carga horária. Conclusão: Exaustão emocional, Despersonalização e Realização profissional atingiram pontuação equivalente a nível médio e alto em grande proporção. A Vitalidade o domínio mais afetado da qualidade de vida. Não houve correlação com carga horária.
\end{abstract}

Palavras-chave: Unidades de Terapia Intensiva; Esgotamento Profissional; Serviço Hospitalar de Fisioterapia; Fisioterapeutas; Estresse Ocupacional.

\section{Abstract}

Background: The Intensive Care Unit is an environment considered stressful, which can affect the quality of life of intensive care physiotherapists and lead to a Burnout Syndrome. Aim: To evaluate the presence of Burnout Syndrome, quality of life and correlation with workload. Methods: Cross-sectional study carried out with 56 physical therapists working in ICUs in the state of Sergipe. Two scales were applied, the Medical Outcomes Short-Form Health Survey that assesses quality of life and the Maslach Burnout Inventory that assesses the Burnout Syndrome, and a sociodemographic questionnaire. Results: The female sex was more prevalent, with $80.3 \%$ of the sample, the mean age was $31.6 \pm 5.6$ years. In terms of quality of life, the most affected domain was Pain, with a median of 62.0 points. On the Burnout Syndrome scale, Emotional Exhaustion averaged $28.9 \pm 5.9$ points with a high-level score in $62.5 \%$ of the sample; the average score in Professional achievement was $15.1 \pm 3.7$ points, with a high level of score in $100 \%$ of the sample; and Depersonalization received a mean score of $17.05 \pm 2.9$ with a high level of score, present in $92.8 \%$ of physical therapists. Quality of life and Burnout Syndrome were not correlated with workload. Conclusion: Emotional exhaustion, Depersonalization and Professional achievement reached scores equivalent to medium and high levels in large proportion. Vitality is the most affected domain of quality of life. There was no correlation with workload.

Keywords: Intensive Care Units; Burnout, Professional; Physical Therapy Departament, Hospital; Physical Therapists; Ocupational Stress.

*Autor correspondente: Yago Alves Lima. E-mail: allvesyaago@gmail.com

Estudo realizado em: Universidade Federal de Sergipe, Lagarto, SE, Brasil.

Aprovação ética: CAAE

91598918.7.0000.5546 da Universidade Federal de Sergipe, $n^{\circ} 2.946 .964$ seja corretamente citado e de forma que não indique endosso ao trabalho feito. Adicionalmente, qualquer trabalho derivado deverá ser publicado sob a mesma licença. 


\section{INTRODUÇÃO}

A Unidade de Terapia Intensiva (UTI) é um setor destinado ao atendimento de pacientes graves ou de risco e dispõe de assistência ininterrupta, com profissionais qualificados, equipamentos específicos, recursos humanos especializados, além de acesso a outras tecnologias destinadas ao diagnóstico e terapêutica' ${ }^{1}$. Neste ambiente é necessária atuação multiprofissional, com equipe dispondo de médicos, enfermeiros, fisioterapeutas, nutricionistas, dentre outros².

A fisioterapia está presente em vários segmentos do tratamento intensivo, como no atendimento a pacientes críticos que necessitem ou não de suporte ventilatório e na assistência durante a recuperação pós-cirúrgica, para assim evitar complicações respiratórias e motoras, que poderão gerar um déficit funcional².

Entre os ambientes hospitalares, a UTI é definida como agressiva e ameaçadora, com base no risco de morte do paciente. Dada a rotina estabelecida em regime de plantão, com alta carga horária de trabalho e de diversas situações emergenciais e concentração de pacientes críticos com alterações súbitas no estado de saúde, o local de trabalho destaca-se como estressante e emocionalmente danoso para a equipe multiprofissional e familiares de pacientes. Os profissionais de saúde, especialmente fisioterapeutas, fazem parte de um grupo de profissionais expostos a estados de tensão excessiva e prolongada: frequentes situações de emergência, inúmeras condições de risco e circunstâncias que propiciam a necessidade constante do acerto, sendo esses alguns dos fatores que justificam a grande probabilidade de estresse ocupacional da classe ${ }^{3,4}$.

O estresse ocupacional ocorre quando o indivíduo não consegue atender às demandas solicitadas pelo seu trabalho, causando sofrimento psíquico, mal-estar, mudanças de comportamento, distúrbios do sono e sentimentos negativos ${ }^{5}$. Para a abordagem do estresse ocupacional, são analisadas as vertentes biológica, psicológica e social, que são complementares e estão interligadas. Na biológica, o estresse caracteriza-se pelo grau de desgaste do corpo. Os processos afetivos, emocionais e intelectuais do indivíduo reportam à abordagem psicológica, ou seja, é a maneira pela qual ele se relaciona com as outras pessoas e com o mundo ao seu redor. A social refere-se à compreensão de como o indivíduo se insere na sociedade. Para diagnosticar o estresse ocupacional, é necessário um estudo clínico dos sinais e sintomas, baseado na investigação individual e nos riscos das situações de trabalho ${ }^{6}$.

Devido à alta morbidade de pacientes, o trabalho nas UTIs é especialmente estressante. Este, quando presente de forma excessiva, possui efeitos deletérios, gerando sensação de sobrecarga, podendo resultar em insônia, fadiga, irritabilidade, ansiedade e depressão, afetando diretamente a sua qualidade de vida dos profissionais da terapia intensiva7,8. Essa tensão física e mental é o que leva a Síndrome de Burnout ${ }^{9}$.
A Síndrome de Burnout, leva em consideração as características individuais associadas as do ambiente e as do trabalho, favorecendo ao aparecimento dos fatores multidimensionais da síndrome: exaustão emocional, esgotamento físico e mental, despersonalização, tratamento impessoal com usuários, insatisfação profissional, sentimento de incompetência e baixa autoestima, levando o profissional ao adoecimento e, muitas vezes, ao afastamento do seu local de trabalho ${ }^{10}$. É importante ressaltar que essa Síndrome foi oficializada pela Organização Mundial da Saúde (OMS) e estará na nova Classificação Internacional de Doenças (CID-11) ${ }^{11}$. Alguns estudos têm estudado a associação entre fatores como idade, sexo, estado civil e carga horária de trabalho e a Síndrome de Burnout, porém ainda não há consenso sobre esta associação ${ }^{10}$.

A maioria dos estudos feitos sob esta temática no ambiente de terapia intensiva são voltados para as áreas de enfermagem e medicina e, apesar do crescimento da atuação fisioterapêutica nas UTIs brasileiras, até o presente momento, não houve nenhum estudo sobre o tema no Estado de Sergipe.

Por isso, este estudo tem como objetivo avaliar a Síndrome de Burnout e qualidade de vida em fisioterapeutas que trabalham em UTIs e correlacionar as variáveis dos questionários com a carga horária semanal de trabalho.

\section{MÉTODOS}

O presente estudo foi aprovado com o seguinte com o seguinte CAAE 87944318.0.0000.8123 pelo Comitê de Ética e Pesquisa da Universidade Federal de Sergipe, $n^{\circ}$ 2.946.964. Trata-se de um estudo de caráter transversal, realizado com fisioterapeutas do estado de Sergipe que há pelo menos 6 meses atuem em UTIs. Inicialmente houve o contato com os coordenadores de fisioterapia e/ou responsáveis técnicos de todas as UTI do Estado de Sergipe, sendo obedecido o protocolo de cada instituição para que fosse possível a realização da coleta de dados. Foi realizado também o contato com o Núcleo de Educação Permanente ou órgão equivalente de cada hospital, sendo a autorização concedida pelo responsável técnico de Fisioterapia da UTI.

Os questionários foram entregues pessoalmente nas UTIs de dez hospitais. A entrega e devolução dos questionários aos fisioterapeutas foram feitas por intermédio dos coordenadores dos serviços. Os fisioterapeutas participantes assinaram o termo de consentimento livre e esclarecido.

A coleta se baseou na aplicação de duas escalas e um questionário sociodemográfico elaborado pelos pesquisadores. As escalas utilizadas foram O Medical Outcomes Short-Form Health Survey (SF-36) que avalia qualidade de vida (QV) ${ }^{12}$ e o Maslach Burnout Inventory (MBI) que avalia as dimensões da síndrome de Burnout ${ }^{13}$. Ambas as escalas são traduzidas e validadas para o Brasil14,15. 
O questionário elaborado conteve os seguintes dados sociodemográficos e profissionais: sexo, idade, estado civil, carga horária semanal, tipo da empresa (pública ou privada), número de vínculos empregatícios, remuneração, tempo de experiência em UTI, participação em eventos e apresentação de trabalhos, titulação de especialista e nível de pós-graduação.

O SF-36, contém 11 questões e 36 itens, e avalia a qualidade de vida de forma genérica em oito domínios, sendo eles: Capacidade funcional, Aspectos físicos, Dor, Estado geral de saúde, Vitalidade, Aspectos sociais, Aspectos emocionais e Saúde mental. A pontuação foi calculada manualmente numa escala de 0 a 100 pontos, na qual 0 é a pior qualidade de vida e 100 é a melhor ${ }^{12}$.

Por último, utilizou-se o MBI, que avalia em 22 questões as três dimensões da Síndrome de Burnout: Exaustão emocional, Despersonalização e Realização profissional ${ }^{2}$. Cada questão é respondida numa escala Likert de sete pontos de acordo com a frequência com que ela acontece. A pontuação obtida em cada dimensão é classificada como nível alto, médio ou baixo de acordo com um valor de corte pré-estabelecido. Para a Exaustão emocional, o nível alto corresponde a pontuação $\geq 27$; nível médio: $\geq 17$ e $\leq 26$ e nível baixo: $\leq 16$. Para a Despersonalização, alto nível é definido como pontuação $\geq 13$; nível médio: $\geq 7$ e $\leq 12$ e nível baixo: $\leq 6$. Para estas duas dimensões, quanto maior a pontuação, maior o nível do aspecto da Síndrome. Já para a Realização profissional, o escore é reverso: uma maior pontuação indica um menor nível (alto nível: $\leq 31$; médio: $\geq 32$ e $\leq 38$; nível baixo: $\geq 39$ pontos) ${ }^{13}$.

A análise estatística foi realizada através dos programas Excel ${ }^{\circledR}$ e SPSS $\circledast 2$ 23.0, considerando $p<0,05$. Para avaliação da normalidade foi utilizado o teste de Kolmogorov-Smirnov. As dimensões do MBI e do SF-36 foram correlacionadas com carga horária através dos testes de Pearson ou Spearman. A análise descritiva foi realizada por meio de número total, porcentagem, média e desvio padrão ou mediana e, mínimo e máximo em planilha Excel®.

\section{RESULTADOS}

Ao todo, 120 questionários foram entregues e 56 fisioterapeutas devolveram respondidos. Foi predominante o sexo feminino $(80,3 \%)$ e também a prestação de serviço no setor público, com 33 fisioterapeutas (58,9\%). A idade média geral foi de 31,6 $\pm 5,6$ anos, sendo predominante o estado civil solteiro $(55,4 \%)$, e a carga horária semanal média de trabalho foi de $38,7 \pm 16,8$ horas. Mais da metade da amostra afirmou ter dois vínculos empregatícios $(53,6 \%)$ e relatou remuneração mensal entre 2.500 e 5.000 reais $(57,1 \%)$ (Tabela 1).

Quanto ao tempo de experiência profissional em UTI, a faixa mais prevalente foi entre 6 e 10 anos (32,1\%). A maioria absoluta afirmou que participa de eventos científicos da área $(92,8 \%)$, sendo que $44,8 \%$ não apresentam trabalhos nesses eventos. $60,7 \%$ relataram ter especialização (Pós-graduação Lato sensu) e 51,8\% da amostra total têm título de especialista concedido pela Associação Brasileira de Fisioterapia Cardiorrespiratória e Fisioterapia em Terapia Intensiva (Tabela 2).

No questionário SF-36, o domínio mais afetado foi dor com uma mediana de 62,0 pontos, seguido por vitalidade com 62,5 pontos e estado geral de saúde com 66,0 pontos, enquanto os domínios menos afetados foram aspectos físicos e emocionais, com uma mediana de 100 pontos (Tabela 3). No teste de correlação de Spearman não houve associação entre carga horária e os domínios do SF-36.

Com relação ao $\mathrm{MBI}$, o domínio de Exaustão emocional teve média de $28,9 \pm 5,9$ pontos, sendo que $62,5 \%$ da amostra apresentou uma pontuação considerada como nível alto. A média de pontos em realização profissional foi de $15,1 \pm 3,7$ pontos, com $100 \%$ da amostra com pontuação considerada nível alto. A despersonalização teve uma média de $17,05 \pm 2,9$ pontos, com $92,8 \%$ dos fisioterapeutas apresentando uma pontuação classificada como nível alto (Tabela 4). O teste de correlação de Pearson não mostrou associação entre carga horaria de trabalho e a pontuação dos domínios do MBI.

Tabela 1. Dados sociodemográficos da amostra.

\begin{tabular}{|c|c|}
\hline Variável & $n=56$ \\
\hline Sexo Feminino, n (\%) & $45(80,3)$ \\
\hline Idade, anos* & $31,6 \pm 5,6$ \\
\hline \multicolumn{2}{|l|}{ Faixa etária, n (\%) } \\
\hline Até 30 anos & $27(48,2)$ \\
\hline 31-39 anos & $25(44,7)$ \\
\hline $40-49$ anos & $4(7,1)$ \\
\hline \multicolumn{2}{|l|}{ Estado Civil, n (\%) } \\
\hline Solteiro & $31(55,4)$ \\
\hline Casado & $22(39,2)$ \\
\hline Divorciado & $3(5,3)$ \\
\hline Carga Horária Semanal, horas* & $38,7 \pm 16,8$ \\
\hline \multicolumn{2}{|l|}{ Nº de vínculos empregatícios, n (\%) } \\
\hline Um vínculo & $22(39,3)$ \\
\hline Dois vínculos & $30(53,6)$ \\
\hline Três vínculos & $4(7,1)$ \\
\hline \multicolumn{2}{|l|}{ Remuneração mensal, n (\%) } \\
\hline $1000-2500$ reais & $10(17,9)$ \\
\hline $2500-5000$ reais & $32(57,1)$ \\
\hline$>5000$ reais & $14(25)$ \\
\hline \multicolumn{2}{|l|}{ Setor de atuação, n (\%) } \\
\hline Público & $33(58,9)$ \\
\hline Privado & $23(41,1)$ \\
\hline
\end{tabular}

*Os dados estão apresentados como média \pm desvio padrão, exceto quando indicado. 
Tabela 2. Caracterização profissional da amostra.

$\mathrm{n}=56$

Tempo de experiência em UTI*, n (\%)

0-3 anos

4-5 anos

6-10 anos

$11-15$ anos

16 anos ou mais

Participação em eventos científicos da área, n (\%)

Apresentação de trabalhos científicos, n (\%)

Sim

Às vezes

Não

Título de especialista, n (\%)

Nível de pós-graduação, n (\%)

Residência

Mestrado

Doutorado

Especialização

$34(60,7)$

*UTI: Unidade de Terapia Intensiva.

Tabela 3. Pontuações obtidas no Medical Outcomes Short-Form Health Survey (SF36).

\begin{tabular}{lccc}
\hline \multicolumn{1}{c}{ Domínio } & Mediana** & Mínimo** & Máximo** \\
\hline Capacidade funcional* & 95,0 & 55,0 & 100,0 \\
Aspectos Físicos* & 100,0 & 0,0 & 100,0 \\
Dor* & 62,0 & 22,0 & 100,0 \\
Estado geral de saúde* & 66,0 & 27,0 & 100,0 \\
Vitalidade* & 62,5 & 15,0 & 95,0 \\
Aspectos sociais* & 75,0 & 25,0 & 100,0 \\
Aspectos emocionais* & 100,0 & 0,0 & 100,0 \\
Saúde mental* & 76,0 & 40,0 & 100,0 \\
Mediana geral & $\mathbf{7 2 , 9}$ & - & - \\
\hline
\end{tabular}

*A pontuação para cada item varia de 0 a 100, sendo que 0 corresponde a pior e 100 a melhor qualidade de vida; **Os dados foram apresentados como mediana, valor mínimo e máximo.

\section{DISCUSSÃO}

Nossos resultados mostraram que os fisioterapeutas atuantes em UTIs do estado do Sergipe apresentaram pontuação elevada nas três dimensões do MBI. A dimensão Exaustão emocional apresentou nível alto de pontuação em $62,5 \%$ da amostra, assim como Realização profissional e Despersonalização, que obtiveram também nível alto de em, respectivamente, 100 e 92,8\% da amostra.
Tabela 4. Pontuação obtida nas três dimensões do Maslach Burnout Inventory (MBI) e a classificação nos níveis alto, médio e baixo de acordo com a pontuação para cada dimensão.

\begin{tabular}{lc}
\hline \multicolumn{1}{c}{ Dimensões do Maslach Burnout Inventory } & $\mathbf{n}=\mathbf{5 6}$ \\
\hline Exaustão emocional, pontuação* & $29,0 \pm 6,0$ \\
Nível alto, $\mathrm{n}(\%)$ & $35(62,5)$ \\
Nível médio, n (\%) & $20(35,7)$ \\
Nível baixo, $\mathrm{n}(\%)$ & $1(1,8)$ \\
Despersonalização, pontuação* & $17,0 \pm 2,9$ \\
Nível alto, n (\%) & $52(92,8)$ \\
Nível médio, $\mathrm{n}(\%)$ & $4(7,2)$ \\
Nível baixo, $\mathrm{n}(\%)$ & 0 \\
Realização profissional, pontuação* & $15,2 \pm 3,7$ \\
Nível alto, $\mathrm{n}(\%)$ & $56(100)$ \\
Nível médio, $\mathrm{n}(\%)$ & 0 \\
Nível baixo, $\mathrm{n}(\%)$ & 0 \\
\hline
\end{tabular}

*Os dados estão apresentados como média \pm desvio padrão, exceto quando indicado. A classificação em nível alto, médio e baixo de acordo com a pontuação obtida em cada dimensão da Maslach Burnout Inventory e segue o seguinte padrão: Para Exaustão Emocional: nível alto: $\geq 27$ pontos; médio: $\geq 17$ e $\leq 26$; baixo: $\leq 16$; para Despersonalização: nível alto: $\geq 13$ pontos; médio: $\geq 7$ e $\leq 12$; baixo: $\leq 6$ e Realização Profissional, que possui escore reverso, nível alto: $\leq 31$ pontos; médio: $\geq 32$ e $\leq 38$; nível baixo: $\geq 39$ pontos.

Os domínios mais afetados no SF-36 foram Vitalidade, seguida por Estado geral de saúde e Dor. O domínio menos afetado foi Capacidade funcional, que avaliou as limitações relativas à capacidade física. Porém, nossos achados não mostraram correlação entre a pontuação destas duas escalas e a carga horária de trabalho.

O perfil sociodemográfico no presente estudo identifica uma população predominante de fisioterapeutas do sexo feminino com uma idade média geral de 31,6 anos, caracterizando uma amostra jovem, na qual a maioria possui estado civil solteiro. A baixa média de idade pode ser explicada pelo fato de ser uma profissão com pouco tempo de regulamentação. Apesar de a maior parte da nossa amostra ser jovem e possuir menos de 10 anos de experiência em UTI, constatamos pontuações elevadas na $\mathrm{MBI}$, revelando que a Síndrome de Burnout pode estar presente mesmo em indivíduos em início de carreira. Um fato a ser ressaltado é que $25 \%$ da nossa amostra relatou tempo de experiência de até 3 anos.

Um estudo realizado com fisioterapeutas dos Estados Unidos da American Physical Therapy Association ${ }^{16}$ mostrou que a média de experiência direta na UTI foi de 7,8 anos, estando na mesma faixa dos fisioterapeutas da nossa pesquisa. Já na Índia, $82 \%$ da amostra relatou menos que 5 anos de experiência em UTI, 78\% dos fisioterapeutas entrevistados tinham entre 20 e 30 anos

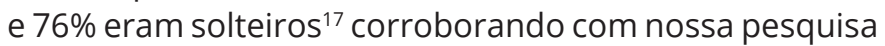
e demonstrando a jovialidade deste público não apenas no Brasil mas também em outros países. 
Com relação ao setor econômico dos hospitais, em um estudo realizado nacionalmente no Brasil, o serviço de fisioterapia esteve mais presente no setor privado compondo $56,4 \%$ da amostra ${ }^{18}$, diferentemente dos nossos achados, o que pode ser atribuído à predominância de hospitais privados no território nacional e a uma melhor estruturação hierárquica do serviço naqueles hospitais, que pode ter propiciado melhor retorno dos questionários aplicados quando comparado ao nosso estudo. Uma pesquisa realizada na cidade de Salvador-BA também difere da amostra do presente estudo, apontando que os hospitais privados foram maioria em relação ao oferecimento de serviços fisioterapêuticos ${ }^{9}$.

A Associação Brasileira de Fisioterapia Cardiorrespiratória e Fisioterapia em Terapia Intensiva (ASSOBRAFIR), através da RDC $n^{\circ} 7$, de 08 de fevereiro de $2010^{19}$, recomenda que os fisioterapeutas das unidades sejam especialistas na área e os coordenadores de fisioterapia tenham o título de especialista concedido pela ASSOBRAFIR. Mais da metade da nossa amostra relatou possuir especialização, e $71 \%$ dos fisioterapeutas brasileiros em ação possuem especializações na área ${ }^{18}$, percebendo-se claramente a preocupação de tornar mais qualificada a formação do fisioterapeuta para atuação em terapia intensiva dado o perfil clínico do paciente nesta área, que requer aperfeiçoamento profissional permitindo maior segurança nas suas atividades laborais.

Além disso, a maioria absoluta dos fisioterapeutas sergipanos que preencheram nosso questionário frequenta eventos da área (93\%), uma proporção superior à relatada em outros estudos. Em um estudo conduzido na Índia, por exemplo, esta proporção foi de $81 \%{ }^{17}$.

Sabe-se que o estresse no trabalho decorre de um desequilíbrio entre as demandas exigidas e a capacidade de supri-las ${ }^{7}$. A busca por capacitação pode melhorar a capacidade do profissional para o enfrentamento das demandas do trabalho, evitando o surgimento de estresse. No entanto, apesar de a maior parte da nossa amostra possuir especialização na área de UTI e participar de eventos científicos da área, os participantes apresentaram altos níveis na pontuação da escala de Síndrome de Burnout.

Em nossa amostra, a faixa de carga horária semanal mais prevalente foi a de 30 a 60 horas. A Resolução $n^{\circ}$ 8.856, de 01/03/1994 do COFFITO traz que o profissional deve ter no máximo 30 horas semanais no ambiente de trabalho ${ }^{20}$. Tal resultado pode ser explicado pelo fato de mais da metade afirmar ter dois vínculos empregatícios recebendo entre 2.500 a 5.000 reais. Mais de um vínculo empregatício indica que os profissionais buscam uma maior remuneração. Porém, o aumento da jornada de trabalho pode aumentar o cansaço físico e mental, reduzir o tempo de lazer e de sono e prejudicar relações pessoais. A carga horária excessiva de trabalho é citada como uma das fontes subjetivas de estresse ${ }^{13}$.

Apesar de nossos dados não terem mostrado associação entre a pontuação do SF-36 e a carga horária de trabalho, os resultados moderados de qualidade de vida obtidos em nossa amostra podem estar relacionados ao desgaste físico e à alta carga horária de trabalho, que os deixam mais exaustos, além de trabalharem em mais de um local, interferindo nas outras atividades do dia a dia ${ }^{21}$.

Em um estudo realizado com profissionais de terapia intensiva da região do Submédio do São Francisco, no Nordeste brasileiro, profissionais com menor carga horária eram mais ativos e apresentaram melhores pontuações nos domínios do SF-36 quando comparados com profissionais com maiores jornadas de trabalho e inativos fisicamente, o que é explicado pelo fato de menores jornadas de trabalho permitir tempo livre para realização de exercícios físicos e consequentemente melhorar a sua qualidade de vida22.

Estudos que avaliam qualidade de vida em fisioterapeutas intensivistas são escassos na literatura. Apenas uma pesquisa, realizada em 2017 por Nascimento et al. ${ }^{23}$ na Bahia, foi específica para fisioterapeutas intensivistas e relatou bons níveis de qualidade de vida nos domínios Físico, Psicológico e Relação social. Esses resultados aproximam-se do que foi encontrado na nossa pesquisa apesar de terem sido obtidos por meio de um questionário distinto, o WHOQOL-bref.

O estudo de Nascimento et al. ${ }^{23}$ avaliou o risco de desenvolvimento do Burnout numa população composta predominantemente por mulheres, com mais de um vínculo empregatício e carga horária excessiva de trabalho, encontrando altos níveis apenas na pontuação da dimensão Realização profissional. Apenas um profissional apresentou a síndrome, porém o estudo ressaltou que uma parcela de profissionais apresentou alto risco de desenvolvê-la, constituindo-se como alerta, já que se não forem implementadas medidas preventivas, estes profissionais poderão vir a desenvolver a síndrome ${ }^{23}$. Esta mesma observação pode ser traçada a partir de um estudo realizado nas cidades de Primavera do Leste e Cuiabá, em Mato Grosso, que mostrou que a maioria dos participantes se encontrava em estágios iniciais da síndrome de Burnout ${ }^{24}$. Já um estudo realizado na cidade de Porto Alegre-RS com profissionais intensivistas encontrou um alto percentual de Burnout moderado e alto ${ }^{25}$.

Um estudo comparou a prevalência da Síndrome de Burnout entre trabalhadores de UTI e fora da UTI, evidenciando que a UTI tem sido associada a uma maior prevalência da síndrome, devido a maior intensidade, carga horária de trabalho e maior grau de dificuldade em relação ao paciente ${ }^{26}$.

Apesar de nossos achados não terem mostrado correlação entre carga horária semanal de trabalho e a pontuação nas escalas de qualidade de vida e de Burnout em fisioterapeutas que trabalham em UTIs, isso não significa que uma carga horária alta não seja prejudicial. Um estudo executado com anestesiologistas em Maceió-AL também não encontrou qualquer correlação entre essas duas variáveis, porém, os autores discutem que dedicação 
em excesso e falta de tempo com a família são queixas muito frequentes e que, associadas a outras condições de trabalho, configuram um ambiente maléfico para a saúde ${ }^{27}$.

Outra pesquisa efetuada no mesmo estado supracitado identificou relação entre as duas variáveis aqui discutidas quando analisadas em médicos plantonistas, sendo observada correlação da carga horária com o domínio Realização profissional do $\mathrm{MBl}^{28}$. Porém, possivelmente, outros fatores além da carga horária de trabalho influenciam na ocorrência da Síndrome de Burnout.

Por interferir na saúde física e mental dos trabalhadores, o estresse ocupacional faz parte de uma importante questão de saúde mundial. Ele interfere na produtividade de suas atividades e até mesmo em afastamento laboral, caracterizando assim o Burnout ${ }^{10}$. O absenteísmo vem aumentando nos últimos anos no Brasil, prejudicando diretamente trabalhador, empresa e paciente. Nosso estudo aponta para uma grande proporção de fisioterapeutas com altos níveis de pontuação na escala de Síndrome de Burnout, embora seja uma população jovem. Seria muito importante elucidar em pesquisas futuras o que esses profissionais usam como estratégia para lidar com o estresse no seu cotidiano e como reduzir a ocorrência deste fenômeno.

O presente trabalho apresentou limitações, dentre elas a baixa adesão dos participantes e o viés de seleção, por se tratar de estudo com aplicação de questionário de participação voluntária. Foram enviados instrumentos de coleta de dados a todos os fisioterapeutas atuantes na área do Estado, mas não houve retorno da população inteira, o que limita a generalização dos resultados.

\section{CONCLUSÃO}

O estudo concluiu que as variáveis Exaustão emocional, Despersonalização e Realização profissional atingiram pontuação considerada média a alta em grande proporção dos participantes, ultrapassando 90\% da amostra, demonstrando uma população em estágios iniciais ou com alta probabilidade de desenvolver a Síndrome de Burnout. Na avaliação da qualidade de vida, o domínio mais afetado foi Dor, seguido por Vitalidade e Estado geral de saúde. Não foi encontrada com a correlação com a carga horária de trabalho.

\section{FONTE DE FINANCIAMENTO}

Nada a declarar.

\section{CONFLITO DE INTERESSES}

Nada a declarar.

\section{REFERÊNCIAS}

1. Loiola NSR, Soares GL, Gonçalves ADS. O papel de um enfermeiro de uma unidade de terapia intensiva na hemodiálise. Rev Uningá. 2017;31(1):40-4.

2. Alves AN. A importância da atuação do fisioterapeuta no ambiente hospitalar. Ens Cienc. 2014;16(6):173-84.

3. Barth AA, Weigel BD, Dummer CD, Machado KC, Tisott TM. Estressores em familiares de pacientes internados na unidade de terapia intensiva. Rev Bras Ter Intensiva. 2016;28(3):323-9. PMid:27737424.

4. Cabral JVB, Neves SC, Oliveira FHPC. Estresse dos profissionais de enfermagem em Unidade de Terapia Intensiva (UTI). Diálogos Acad. 2016;11(2):33-42.

5. Dias FM, Santos JFC, Abelha L, Lovisi GM. O estresse ocupacional e a síndrome do esgotamento profissional (burnout) em trabalhadores da indústria do petróleo: uma revisão sistemática. Rev Bras Saúde Ocup. 2016;41(11):1-12. http://dx.doi.org/10.1590/2317-6369000106715.

6. Prado CEP. Estresse ocupacional: causas e consequências. Rev Bras de Medicina do Trabalho. 2016;14(3):285-9. http:// dx.doi.org/10.5327/Z1679-443520163515.

7. Santos CLC, Nascimento CL So, Barbosa GB. Síndrome de Burnout em fisioterapeutas intensivistas: uma revisão sistemática. Rev Pesq Fisioter. 2017;7(1):103-14. http:// dx.doi.org/10.17267/2238-2704rpf.v7i1.1099.

8. WHO: World Health Organization [Internet]. Burn-out na "occupational phenomenon: International Classification of Diseases. Switzerland: WHO; 2019 [citado em 2019 Out 27]. Disponível em: https://www.who.int/mental_health/ evidence/burn-out/en/

9. Pimenta FA, Simil FF, Tôrres HO, Amaral CF, Rezende CF, Coelho TO, et al. Avaliação da qualidade de vida de aposentados com a utilização do questionário SF-36. Rev Assoc Med Bras. 2008;54(1):55-60. http://dx.doi. org/10.1590/S0104-42302008000100021. PMid:18392487.

10. Silva RAD, Araújo $B$, Morais CCA, Campos SL, Andrade AD, Brandão DC. Síndrome de Burnout: realidade dos fisioterapeutas intensivistas? Rev Fisioter Pesq. 2018;25(4):388-94. http://dx.doi.org/10.1590/18092950/17005225042018.

11. Ciconelli RM, Ferraz MB, Santos W, Meinão I, Quaresma MR. Tradução para a língua portuguesa e validação do questionário genérico de avaliação de qualidade de vida SF-36 (Brasil SF-36). Rev Bras Reumatol. 1999;39(3):143-50.

12. Gonzaga LA. Validação do Maslach Burnout Inventory em Língua Portuguesa: uma investigação estatística sobre a síndrome de Burnout [dissertação]. São Paulo: Programa de Pós-graduação em Administração de Empresas, UniFecap; 2003.

13. Nozawa E, Sarmento GJV, Vega JM, Costa D, Silva JEP, Feltrim MIZ. Perfil de fisioterapeutas brasileiros que atuam em unidades de terapia intensiva. Rev Fisioter Pesq. 2008;15(2):177-82. http://dx.doi.org/10.1590/S180929502008000200011.

14. Brasil. Agência Nacional de Vigilância Sanitária. Resolução $\mathrm{n}^{\circ} 7$ de 24 de fevereiro de 2010. Diário Oficial da União; Brasília; 2010.

15. Santos LFO, Chahud KDH, Gomes M No, Almeida MLO. Perfil dos fisioterapeutas que atuam em unidades de terapia intensiva na cidade de Salvador/Bahia [trabalho de conclusão da pós-graduação em fisioterapia hospitalar]. Salvador: Escola Bahiana de Medicina e Saúde Pública; 2014 [citado em 2019 Out 27]. Disponível em: http://www7. bahiana.edu.br/jspui/handle/bahiana/300 
16. Yeole UL, Chand AR, Nandi BB, Gawali PP, Adkitte RG. Physiotherapy practices in Intensive Care Units across Maharashtr. Indian J Crit Care Med. 2015;19(11):66973. http://dx.doi.org/10.4103/0972-5229.169346. PMid:26730119.

17. Brasil. Lei n 8856 de 01 de março de 1994. Diário Oficial da União; Brasília; 1994.

18. Malone D, Ridgeway K, Nordon-Craft A, Moss P, Schenkman $M$, Moss M. Physical therapist practice in the intensive care unit: results of a national survey. Phys Ther. 2015;95(10):133544. http://dx.doi.org/10.2522/ptj.20140417. PMid:26045604.

19. Moss M, Good VS, Gozal D, Kleinpell R, Sessler CN. An official critical care societies collaborative statement: Burnout syndrome in critical care healthcare professionals: a call for action. Crit Care Med. 2016;44(7):1414-21. http://dx.doi. org/10.1097/CCM.0000000000001885. PMid:27309157

20. Losa Iglesias ME, Bengoa Vallejo RB. Prevalence and relationship between Burnout, job satisfaction, stress, and clinical manifestations in Spanish critical care nurses. Dimens Crit Care Nurs. 2013;32(3):1307. http://dx.doi. org/10.1097/DCC.0b013e31828647fc. PMid:23571196.

21. Almeida LA, Medeiros IDS, Barros AG, Martins CCF, Santos VEP. Fatores geradores da síndrome de Burnout em profissionais da saúde. Rev Fund Care Online. 2016;8(3):4623-8.

22. Freire $C B$, Dias RF, Schwingel PA, França EET, Andrade FMD, Costa EC, et al. Qualidade de vida e atividade física em profissionais de terapia intensiva do sub médio São Francisco. Rev Bras Enferm. 2015;68(1):26-31. http://dx.doi. org/10.1590/0034-7167.2015680104p. PMid:25946491.
23. Nascimento CP, Miranda VC, Ferreira JB, Moraes KCS. Síndrome de Burnout em fisioterapeutas intensivistas. Rev Pesq Fisioter. 2017;7(2):188-98. http://dx.doi. org/10.17267/2238-2704rpf.v7i2.1302.

24. Carvahais FR, Aguilar AMM, Mendonça RL, Ottano C. Frequência da Síndrome de Burnout em unidade de terapia intensiva: uma perspectiva multiprofissional. Rev Pre de Infec e Saúde. 2015;1(4):1-10. http://dx.doi.org/10.26694/ repis.v1i4.4271.

25. Timenetsky KT, Castro CA, Eid RC, Carnieli-Cazati D. Prevalence of burnout syndrome among physiotherapist working in intensive care units and stepdown unit. Intensive Care Med Exp. 2015;3(Supl 1):A722. http://dx.doi. org/10.1186/2197-425X-3-S1-A722.

26. Hoppen CMS, Kissmann N, Chinelato JR, Coelho VP, Wenczenovicz $C$, Nunes $F C L$, et al. High prevalence of burnout syndrome among intensivists of the city of Porto Alegre. Rev Bras Ter Intensiva. 2017;29(1):115-20. http:// dx.doi.org/10.5935/0103-507X.20170017. PMid:28444081.

27. Barbosa FT, Eloi RJ, Santos LM, Leão BA, Lima FJC, Souza-Rodrigues CF. Correlação entre a carga horária semanal de trabalho com a síndrome de burnout entre médicos anestesiologitas de Maceió-AL. Rev Bras Anestesiol. 2017;67(2):115-21. http://dx.doi.org/10.1016/j. bjan.2015.06.001. PMid:27062888.

28. Novais RN, Rocha LM, Eloi RJ, Santos LM, Ribeiro MV, Ramos FW, et al. Prevalência da síndrome de burnout em cirurgiões plantonistas de um hospital de referência para trauma e sua correlação com carga horária semanal de trabalho: estudo transversal. Rev Col Bras Cir. 2016;46(5):314-9. PMid:27982323. 